\title{
Thermoelectric effect in single layer epitaxial graphene formed on semiconductor substrate. Simple analytical model
}

\author{
Z.Z. Alisultanov \\ A.M. Prokhorov General Physics Institute of RAS, 38 Vavilov Str., Moscow 119991, Russia \\ Amirkhanov Institute of Physics of Daghestan Scientific Center of RAS \\ 94 Yaragskovo Str., Makhachkala 367003, Russia \\ Dagestan State University, 43-a Gadzhiyev Str., Makhachkala 367000, Russia \\ E-mail: zaur0102@gmail.com
}

Received December 27, 2012, revised February 22, 2013

\begin{abstract}
In this paper we have investigated thermoelectric effect in the epitaxial graphene on a semiconductor substrate using a simple model. We have obtained the expressions for static conductance and thermopower of the epitaxial graphene. The thermopower of the epitaxial graphene can be abnormally large near the edges of the semiconductor band gap.
\end{abstract}

PACS: 68.43.-h Chemisorption/physisorption: adsorbates on surfaces;

72.80.Vp Electronic transport in graphene;

65.80.Ck Thermal properties of graphene.

Keywords: graphene, thermopower.

\section{Introduction}

Graphene, a two-dimensional allotrope of carbon, has a unique band structure, in which electrons are described by linear and gapless dispersion law [1]. Unusual spectrum and geometry of the lattice lead to the unique transport properties of graphene [1-3]. Effects of various factors (impurities, vacancies, substrate, etc.) on the electronic spectrum of graphene was investigated partially in [4-6]. The study of epitaxial graphene (EG) is one of the main problems in the physics of graphene [6-9]. The properties of EG sheets are of interest for several reasons. First of all, graphene on the surface of metals and semiconductors can be considered as an effective contact for devices [10]. On the other hand, in order to make full use of the properties of graphene in electronics, it is necessary to vary the structure, chemical composition, morphology, etc., that can be done using a suitable substrate.

The study of thermoelectric properties provides complementary information to the electronic structure of condensed matter. The Mott formula [11] is the main expres- sion in the theory of thermoelectric effect. This formula connects the differential thermopower with the logarithmic derivative of the longitudinal conductivity

$$
S_{x x}=-\frac{\pi^{2}}{3 e} k_{B} T \frac{d}{d \mu}[\ln \sigma(\mu, T=0)],
$$

where $T$ is temperature, $\sigma(\mu, T=0)$ denotes conductance at zero temperature, $\mu$ denotes chemical potential, $k_{B}$ is the Boltzmann constant.

Graphene has unique thermoelectric properties (see, e.g., review [12]). In fact, the thermopower of graphene at room temperature reaches about $100 \mathrm{mV} / \mathrm{K}$ (for comparison, the thermopower of metals is about $0.01 \mathrm{mV} / \mathrm{K}$ ). Such large values of the thermoelectric power were observed in gapped graphene, when the chemical potential is close to the edge of the band gap. In this paper, using a simple model we investigated thermoelectric effect in the EG, which formed on a semiconductor substrate. 


\section{Model of epitaxial graphene}

We investigate EG using the model which was proposed by Davydov [13]. In [14-18], this model was applied to different cases: EG on metal, EG on semiconductor, EG on size-quantized film. In this model, the EG is represented as carbon atoms adsorbed on the substrate and arranged in a hexagonal structure [13]. In the Anderson model for the Green function of single adatom, we have

$$
G_{i}^{0}=\left(\varepsilon-\varepsilon_{a}-\Lambda(\varepsilon)-i \Gamma_{c}(\varepsilon)\right)^{-1},
$$

where $\varepsilon_{a}$ is the energy of adatom level, $\Gamma_{c}(\varepsilon)=$ $=\pi|V|^{2} \rho(\varepsilon)$ denotes the half-width of adatom level, $\Lambda(\varepsilon)=\frac{1}{\pi} P \int_{-\infty}^{\infty} \Gamma_{c}\left(\varepsilon^{\prime}\right) d \varepsilon^{\prime} /\left(\varepsilon-\varepsilon^{\prime}\right)$ is the level shift function, $\rho(\varepsilon)$ is the density of states of substrate, " $P$ " denotes the principle value of the integral. Between carbon adatoms arranged in a hexagonal structure occurs electron exchange. Consequently, Brillouin zone is formed in the adsorbed layer. To determine the Green's function of the perturbed system, we use the Dyson equation:

$$
G_{i j}=G_{i}^{0} \delta_{i j}+\sum_{l} G_{i}^{0} t_{i l} G_{l j},
$$

where $t_{i l}=t$ is the nearest-neighbor hopping energy (for isolated graphene $t \approx 2.8 \mathrm{eV}$ ). Applying Fourier transform to Dyson equation, given the symmetry of the crystal lattice of graphene, and given that $G_{i}^{0}=G_{0}$, we get

$$
G_{\boldsymbol{q}}^{-1}(\varepsilon)=G_{0}^{-1}(\varepsilon)-t f(\mathbf{q})-i \gamma
$$

where

$$
f(\mathbf{q})=v_{b} \sqrt{3+2 \cos \left(\sqrt{3} q_{x} a\right)+4 \cos \left(\sqrt{3} q_{x} a / 2\right) \cos \left(3 q_{y} a / 2\right)},
$$

$a$ is the carbon-carbon distance (for isolated graphene $a \approx 1.42 \AA$ ), $\mathbf{q}$ is the two-dimensional wave vector of the electron, $v_{b}$ is the band index: $v_{b}=+1$ corresponds to the conduction band and $v_{b}=-1$ corresponds to the valence band, $\gamma$ is the energy damping of quasiparticle due to scattering (by phonons, etc.). Near the Dirac point for the energy spectrum, we obtain $t f(\mathbf{k})=v_{b} 3 t a|\mathbf{k}| / 2=v_{b} v_{F}|\mathbf{k}|$, where $\mathbf{k}$ is the wave vector measured relatively to the Dirac points and $v_{F}$ is the Fermi velocity (for electrons in of isolated graphene $v_{F} \approx 10^{8} \mathrm{~cm} / \mathrm{s}$ ). For the density of the states of substrate, we use the Haldane-Anderson model: $\rho(\varepsilon)=\rho_{0} \Theta(|\varepsilon|-\Delta)$, where $\Delta$ is the band gap in the energy spectrum of semiconductor. Then

$$
\begin{gathered}
\Gamma_{c}(\varepsilon)=\pi|V|^{2} \rho_{0} \Theta(|\varepsilon|-\Delta) \\
\text { and } \Lambda(\varepsilon)=\pi|V|^{2} \rho_{0} \ln \left|\frac{\Delta-\varepsilon}{\Delta+\varepsilon}\right| \text {. }
\end{gathered}
$$

\section{Static conductance and thermopower of EG}

Assuming an electric field applied in the $x$ direction, the frequency dependent (real part) conductivity is calculated from the Kubo-Greenwood formula [19]

$$
\begin{aligned}
& \sigma(\omega, T)=-\frac{v_{F}^{2} \hbar^{2} e^{2}}{N S \omega} \int_{-\infty}^{\infty} \frac{d \varepsilon}{2 \pi \hbar}[f(\varepsilon+\omega)-f(\varepsilon)] \times \\
& \times \sum_{k, v_{b 1}, v_{b 2}= \pm 1} \operatorname{Im} G(\mathbf{k}, \varepsilon) \operatorname{Im} G(\mathbf{k}, \varepsilon+\omega),
\end{aligned}
$$

where $f(\varepsilon)=\left(\mathrm{e}^{(\varepsilon-\mu) / T}+1\right)^{-1}$ is the Fermi-Dirac distribution function, $v_{b 1}$ and $v_{b 2}$ are the band indices for $G(\mathbf{k}, \varepsilon)$ and $G(\mathbf{k}, \varepsilon+\omega)$, respectively, $S$ denotes the area of graphene. In (3), the number of bands, valley and the spin degeneracy is taken into account. The KuboGreenwood formula for the static conductance at zero temperature reads

$$
\begin{gathered}
\sigma(0, T=0)=-\frac{e^{2}}{2 \pi^{2} \hbar} \times \\
\times \sum_{v_{b 1}, v_{b 2}= \pm 1} \int_{0}^{\xi} \varepsilon_{k} d \varepsilon_{k} \operatorname{Im} G_{v_{b 1}}\left(\varepsilon_{k}, \mu\right) \operatorname{Im} G\left(\varepsilon_{k}, \mu\right),
\end{gathered}
$$

where $\xi$ is the band width of graphene $(\xi \sim t), \mu$ is the chemical potential of graphene. Performing the integration in (4), we have

$$
\begin{gathered}
\sigma_{G-S}=\frac{e^{2}}{\pi \hbar}\left[\left(\frac{\left(\tilde{\mu}^{2}-\Gamma^{2}\right) F(\Gamma)-4 \tilde{\mu}^{2} \Gamma^{2}}{F^{2}(\Gamma)+4 \tilde{\mu}^{2} \Gamma^{2}}+1\right)+\right. \\
\left.+\frac{1}{2}\left(\frac{\tilde{\mu}}{\Gamma}+\frac{\Gamma}{\tilde{\mu}}\right)\left(\operatorname{arctg} \frac{F(\Gamma)}{2 \tilde{\mu} \Gamma}+\operatorname{arctg} \frac{\tilde{\mu}^{2}-\Gamma^{2}}{2 \tilde{\mu} \Gamma}\right)\right],
\end{gathered}
$$

where $\quad F(\Gamma)=\xi^{2}-\tilde{\mu}^{2}+\Gamma^{2}, \quad \tilde{\mu}=\mu-\Lambda(\mu), \quad \Gamma=\gamma+$ $+\Gamma_{c} \Theta\left(\left|\varepsilon_{F}\right|-\Delta\right)$. The result (5) shows that, when $\gamma, \Gamma_{c} \rightarrow 0$ conductance has a universal value $2 e^{2} / \pi \hbar$ independent of the dilution concentration, in agreement with earlier theoretical works and in agreement with the experimental data on graphene. The dependence of the EG static conductance on the chemical potential is shown in Fig. 1. From Fig. 1 we see that near the edges of the band gap, dependence has a resonant character. We believe, that $\gamma=0.1 \mathrm{eV}, \quad \xi=3 \mathrm{eV}, \quad v_{F}=10^{8} \mathrm{~cm} / \mathrm{s} \quad$ and $\Delta=1.5 \mathrm{eV}$ (for example, for $\mathrm{SiC}$ polytypes $\Delta \approx 1.55 \mathrm{eV}$ ). A resonant character of the conductivity related to the fact that near the edges of the band gap relaxation time of quasiparticles is strongly dependent on energy. This dependence related to the fact that in the vicinity edges of the substrate band gap the band gap opens in spectrum of EG [13,17]. Latter is caused by the fact that in the region of the substrate band gap $\Gamma_{c}=0$, but $\Lambda \neq 0$. It needs to call the readers attention 


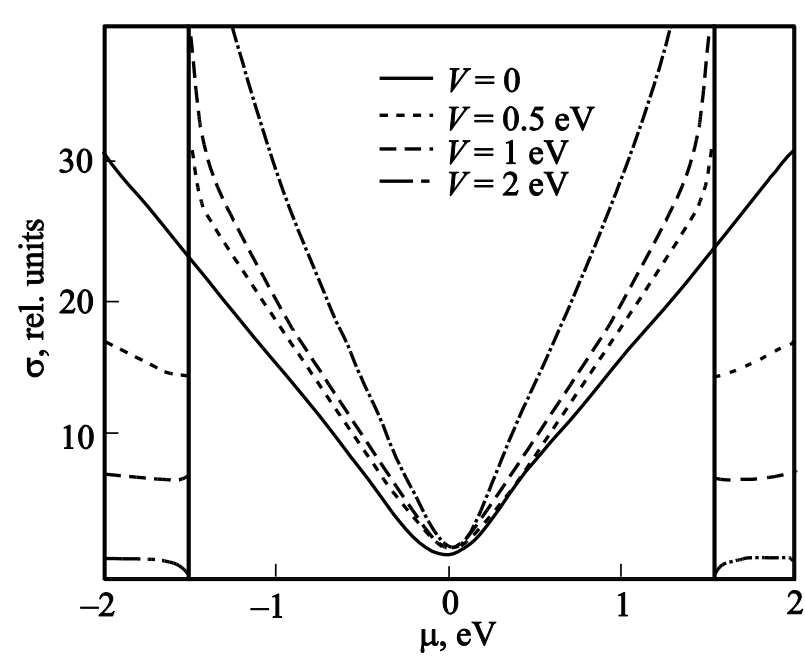

Fig. 1. The conductance (in units of $e^{2} / \pi \hbar$ ) of epitaxial graphene, formed on the semiconductor substrate.

to the evident analogy between the transport in gapped graphene and in metal close to the electronic topological transition [20,21]. Indeed, in the vicinity of the critical point, when the Fermi surface connectivity changes, the quasiparticle relaxation rate also acquires a contribution depending on energy in the form of step function. This generates the well known kinks in conductivity [12,20,21].

A detailed study of the transport properties of epitaxial graphene formed on the semiconductor substrate is given in [22]. In this work, the dependence of static and dynamic conductivity on temperature is also investigated.

Using the formula (1) we obtained the thermopower of EG. The dependence of the EG thermopower on chemical potential at constant temperature is shown in Fig. 2. From Fig. 2 we see that near the edges of the band gap, thermopower is very high. The reason for the increasing of the

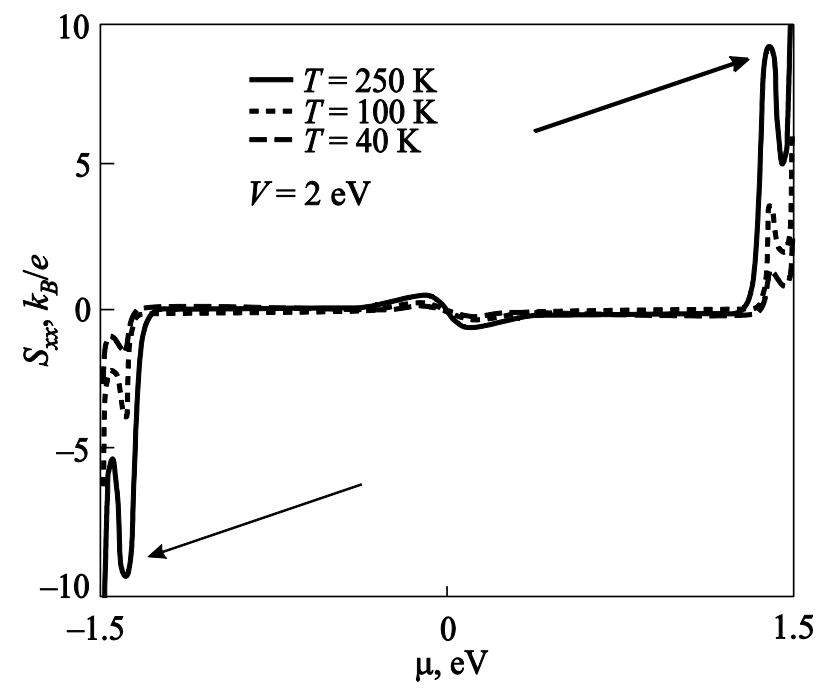

Fig. 2. The dependence of thermopower of epitaxial graphene on chemical potential. thermopower near the edge of the band gap is explained above and in Refs. 12, 20, 21.

To get the dependence of the thermopower on temperature, it is necessary to know the temperature dependence of the chemical potential, which is gives by expression

$$
n=\frac{2}{\pi\left(\hbar \cup_{F}\right)^{2}} \int_{0}^{\infty} \varepsilon[f(\varepsilon-\mu)-f(\varepsilon+\mu)] d \varepsilon,
$$

where $n$ is the carrier concentration. If we use the formula (6), then this leads to a deviation from the linear dependence of the thermoelectric power on the temperature. This conclusion is confirmed by the experimental results [9].

\section{Conclusion}

A simple model of EG used here was first proposed in Ref. 13. Such phenomena as the charge exchange between the graphene and the substrate, the occurrence of a gap in the graphene by indirect interaction of its atoms through the electron gas of the substrate, the occurrence of a gap in the graphene formed on the semiconductor substrate, the effect of external magnetic field on the charge exchange and other phenomena have been investigated by S.Yu. Davydov and Z.Z. Alisultanov [13-18,22]. Realized in these works numerous comparison of analytical results with the experimental data and the data obtained from the first principles, show that the model used is quite adequate and can be used for qualitative calculations.

When designing the novel nanoelectronic devices it will be possible to control their temperature regime using the Peltier cooling effect, which is governed by the value of the thermoelectric coefficient. Thermoelectric power in EG can be tuned by choosing a suitable substrate, what looks promising for practical applications.

Author thanks the Federal Lezghin National and Cultural Autonomy (FLNCA) for the support and A.A. Varlamov for useful discussion.

1. A.H. Castro Neto, F. Guinea, N.M.R. Peres, K.S. Novoselov, and A.K. Geim, Rev. Mod. Phys. 81, 109 (2009).

2. V.P. Gusynin and S.G. Sharapov, Phys. Rev. Lett. 95, 146801 (2005); V.P. Gusynin, S.G. Sharapov, and J.P. Carbotte, Phys. Rev. Lett. 96, 256802 (2006).

3. L.A. Falkovsky, JETP 106, 575 (2008); J. Exp. Theor. Phys. 142, 1309 (2012).

4. V.M. Loktev and Yu.V. Skrypnyk, Phys. Rev. B 73, 241402(R) (2006); Fiz. Nizk. Temp. 33, 1002 (2007) [Low Temp. Phys. 33, 762 (2007)]; Fiz. Nizk. Temp. 34, 1040 (2008) [Low Temp. Phys. 34, 818 (2008)].

5. V.M. Loktev and V. Turkovski, Fiz. Nizk. Temp. 35, 805 (2009) [Low Temp. Phys. 35, 632 (2009)].

6. Z.Z. Alisultanov, Fiz. Nizk. Temp. 39, 225 (2013) [Low Temp. Phys. 39, 172 (2013)]; Fiz. Tverd. Tela 55, 1211 (2013). 
7. C. Berger, Z. Song, T. Li, X. Li, A.Y. Ogbazghi, R. Feng, Z. Dai, A.N. Marchenkov, E.H. Conrad, Ph.N. First, and W.A. de Heer, J. Phys. Chem. B 108, 19912 (2004).

8. C. Faugeras, A. Nerriere, M. Potemski, A. Mahmood, E. Dujardin, C. Berger, and W.A. de Heer, Appl. Phys. Lett. 92, 011914 (2008).

9. X. Wu, Y. Hu, M. Ruan, N.K. Madiomanana, C. Berger, and W.A. de Heer, Appl. Phys. Lett. 99, 133102 (2011).

10. W.J. Lu, W.C. Mitchel, C.A. Thornton, W.E. Collins, G.R. Landis, and S.R. Smith, J. Electrochem. Soc. 150, G177 (2003).

11. N.F. Mott and H. Jones, The Theory of the Properties of Metals and Alloys, 1st ed., Oxford, Clarendon Press (1936).

12. A.A. Varlamov, A.V. Kavokin, I.A. Luk'yanchuk, and S.G. Sharapov, Phys. Usp. 55, 1146 (2012).

13. S.Yu. Davydov, Semiconductors 45, 618 (2011).

14. S.Yu. Davydov, Tech. Phys. Lett. 37, 476 (2011).
15. Z.Z. Alisultanov and R.P. Meilanov, Phys. Solid State 54, 1486 (2012); Theoret. and Math. Phys. 172, 1278 (2012).

16. Z.Z. Alisultanov, S.V. Garnov, and R.P. Meilanov, Fiz. Tverd. Tela 54, 2237 (2012).

17. S.Yu. Davydov, Fiz. i Tekh. Poluprovodnikov 47, 97 (2013).

18. Z.Z. Alisultanov, Tech. Phys. Lett. 39, 23 (2013).

19. N.M.R. Peres, F. Guinea, and A.H. Castro Neto, Phys. Rev. $B$ 73, 125411 (2006).

20. Ya.M. Blanter, M.I. Kaganov, A.V. Pantsulaya, and A.A. Varlamov, Phys. Rep. 245, 159 (1994).

21. S.G. Sharapov and A.A. Varlamov, Phys. Rev. B 86, 035430 (2012).

22. Z.Z. Alisultanov, Physica B (to be published). 